\title{
Hirsutine, an indole alkaloid of Uncaria rhynchophylla, inhibits inflammation-mediated neurotoxicity and microglial activation
}

\author{
HWAN YONG JUNG ${ }^{1 *}$, KYONG NYON NAM $^{2 *}$, BYUNG-CHOEL WOO $^{1}$, \\ KYOO-PIL KIM ${ }^{1}$, SUNG-OK KIM ${ }^{3}$ and EUNJOO H. LEE ${ }^{2}$ \\ ${ }^{1}$ Department of Cardiovascular and Neurologic Diseases (Stroke Center), Hospital of Oriental Medicine, \\ Kyung Hee University, Seoul 130-702; ${ }^{2}$ Graduate School of East-West Medical Science, \\ Kyung Hee University, Yongin-si 446-701; ${ }^{3}$ Department of Herbal Pharmacology, \\ College of Oriental Medicine, Daegu Haany University, Daegu 706-060, Republic of Korea
}

Received July 14, 2012; Accepted October 10, 2012

DOI: $10.3892 / \mathrm{mmr} .2012 .1135$

\begin{abstract}
Chronic microglial activation endangers neuronal survival through the release of various pro-inflammatory and neurotoxic factors. As such, negative regulators of microglial activation have been considered as potential therapeutic candidates to reduce the risk of neurodegeneration associated with inflammation. Uncaria rhynchophylla (U. rhynchophylla) is a traditional oriental herb that has been used for treatment of disorders of the cardiovascular and central nervous systems. Hirsutine (HS), one of the major indole alkaloids of U.rhynchophylla, has demonstrated neuroprotective potential. The aim of the present study was to examine the efficacy of HS in the repression of inflammation-induced neurotoxicity and microglial cell activation. In organotypic hippocampal slice cultures, HS blocked lipopolysaccharide (LPS)-related hippocampal cell death and production of nitric oxide (NO), prostaglandin (PG) $\mathrm{E}_{2}$ and interleukin-1 $\beta$. HS was demonstrated to effectively inhibit LPS-induced NO release from cultured rat brain microglia. The compound reduced the LPS-stimulated production of $\mathrm{PGE}_{2}$ and intracellular reactive oxygen species. HS significantly decreased LPS-induced phosphorylation of the mitogen-activated protein kinases and Akt signaling proteins. In conclusion, HS reduces the production of various neurotoxic factors in activated microglial cells and possesses neuroprotective activity in a model of inflammationinduced neurotoxicity.
\end{abstract}

Correspondence to: Professor Eunjoo H. Lee, Graduate School of East-West Medical Science, Kyung Hee University, 1 Seochun, Yongin-si 446-701, Republic of Korea

E-mail: ehwang@khu.ac.kr

${ }^{*}$ Contributed equally

Key words: brain inflammation, hirsutine, microglia, mitogenactivated protein kinase, neurotoxicity, nitric oxide, organotypic hippocampal slice culture, reactive oxygen species, Uncaria rhynchophylla

\section{Introduction}

Immune and inflammatory responses in the central nervous system (CNS) are principally mediated by microglia. These responses are activated during neuropathological conditions and restore CNS homeostasis (1). The activation of microglia involves proliferation, migration to the injury site, increased expression of immunomodulators and transformation into phagocytes $(1,2)$. Activated microglia also promote neuronal injury through the release of proinflammatory and cytotoxic factors, including cytokines, nitric oxide (NO) and reactive oxygen species (ROS) (2). Chronic microglial activation has been implicated in neuronal destruction associated with various neurodegenerative diseases, including Alzheimer's and Parkinson's (3). Therefore, downregulation of negative-regulatory mechanisms to reduce the activation of microglial cells is essential to avoid excessive CNS inflammatory processes (4). The identification of agents that target over-activated microglial cells is essential for the reduction of neuronal destruction associated with neurodegenerative diseases.

Uncaria rhynchophylla is a traditional oriental herb that has been used for treatment of disorders of the cardiovascular and central nervous systems (5). Hirsutine (HS) is a major indole alkaloid of $U$. rhynchophylla. HS has been reported to have antihypertensive and antiarrhythmic activities through its effects on intracellular $\mathrm{Ca}^{2+}$ levels in rat aorta and the action potential in cardiac muscle $(6,7)$. In a previous study, HS was demonstrated as effective for the protection of rat cardiomyocytes from hypoxia-induced cell death (8). Studies using animal models have revealed that extracts isolated from $U$. rhynchophylla demonstrate neuroprotective potential against diverse neuronal injuries associated with excitotoxicity, amnesia, epileptic seizures and Parkinson's and Alzheimer's disease (9-13). In vitro studies on the neuroprotective roles of HS have demonstrated that the compound attenuates glutamate-induced cell death in PC12 and cerebellar granule cells $(14,15)$. Based on these studies, components of $U$. rhynchophylla have been proposed to act as neuroprotective agents. However, the efficacy of the compounds on neuroinflammation control has been largely unexplored. The purpose of the present study was to examine 
the ability of HS in the control of inflammatory responses of the brain microglia and the protective potential of HS for reducing inflammation-induced neurotoxicity.

\section{Materials and methods}

Drug, chemicals and reagents. All cell and tissue culture products were purchased from Invitrogen (Carlsbad, CA, USA). HS (product no. 082-0461) was purchased from Wako Pure Chemical Industries (Osaka, Japan). Escherichia coli lipopolysaccharide (LPS) and other chemicals were purchased from Sigma (St. Louis, MO, USA). Antibodies against phospho-p44/42 MAPK, p44/42 MAPK, phospho-SAPK/ JNK, SAPK/JNK, phospho-p38, p38, phospho-Akt and Akt were purchased from Cell Signaling Technology (Beverly, MA, USA).

Experimental animals. Rats were maintained in accordance with the Institutional Animal Care and Use Committee guidelines of Kyung Hee University. All animal protocols were approved by the Animal Ethics Committee of Kyung Hee University in accordance with the 14th article of the Korean Animal Protection Law.

Organotypic hippocampal slice culture. Organotypic hippocampal slice cultures were prepared from male SpragueDawley rats (seven days old; Orient, Kyunggido, Korea) using the methods previously described by Stoppini and others $(16,17)$. Briefly, the hippocampus was isolated and cut transversely at a thickness of $350 \mu \mathrm{m}$ with a McIlwain Tissue Chopper (Mickle Laboratory Engineering, Surrey, UK). The slices were placed on membrane inserts (Millicell-CM; Millipore, Bedford, MA, USA) in six-well plates. Each well contained $1 \mathrm{ml}$ of culture medium composed of $50 \% \mathrm{MEM}$, 25\% Hank's Balanced Salt Solution and 25\% horse serum. The slices were cultured at $36^{\circ} \mathrm{C}$ in an incubator in the presence of $5 \% \mathrm{CO}_{2}$ for 12-14 days and the medium was changed every 2-3 days.

LPS treatment and assessment of neuronal damage. Neurotoxicity was evaluated by the uptake of the fluorescent dye propidium iodide (PI) as previously described $(17,18)$. Briefly, LPS $(10 \mu \mathrm{g} / \mathrm{ml})$ was applied to hippocampal cultures with or without pretreatment with HS. Following LPS treatment, the culture medium was collected and subjected to the nitrite assay prior to being replaced with fresh serum-free medium containing $5 \mu \mathrm{g} / \mathrm{ml}$ PI. Neuronal death was observed within 30-60 min of PI addition. PI-stained images were captured using a laser scanning microscope (LSM 510; Carl Zeiss, Cambridge, UK) and the observed PI-uptake areas were measured using confocal microscopy with LSM 510 software (release 3.2; Carl Zeiss). All the data were background subtracted using the fluorescence emission originated from a region on the insert containing no tissue. For immunofluorescent staining of neurons, hippocampal slices were fixed in $4 \%$ paraformaldehyde and stained with Alexa fluor 488-conjugated mouse anti-NeuN monoclonal antibody (Chemicon International, Temecula, CA, USA). The immunostained images were observed under a Carl Zeiss LSM 510 microscope.
Primary microglia culture. Primary microglial cells were prepared from cerebral cortices of one-day-old rat pups (Orient) as described previously $(19,20)$. Cells reached confluence at 12-14 days and flasks were agitated to remove the microglia. The detached cells were incubated for $1 \mathrm{~h}$ and the non-adherent cells were removed. The adherent microglial cells were cultured for $24 \mathrm{~h}$ and the purity of the cultures was routinely $>95 \%$, as judged by immunostaining with an antiOX-42 antibody (Chemicon). The cells were pretreated with HS in fresh medium containing $0.1 \%$ fetal bovine serum for $30 \mathrm{~min}$ prior to the addition of LPS.

Nitrite assay. Nitrite in culture supernatants was measured as an indicator of NO production. An aliquot of the culture supernatant was mixed with a volume of Griess reagent (Molecular Probes, Eugene, OR, USA) and the absorbance at $570 \mathrm{~nm}$ was determined using a microplate reader. Sodium nitrite $(0-100 \mu \mathrm{M})$ was used as a standard to assess nitrite concentrations.

Cell viability assay. For the cell viability assay, cultures were incubated in MTT solution ( $1 \mathrm{mg} / \mathrm{ml}$; Sigma) in two volumes of culture medium for $1 \mathrm{~h}$ at $37^{\circ} \mathrm{C}$. The MTT solution was then removed, the cells were dissolved in dimethyl sulfoxide (150 $\mu \mathrm{l})$ and optical density of the samples was measured at $570 \mathrm{~nm}$ using a microplate reader.

$I L-1 \beta$ and prostaglandin $(P G) E_{2}$ assays. Following each treatment, culture medium was collected in microcentrifuge tubes and centrifuged at $10,000 \mathrm{x}$ g for $10 \mathrm{~min}$. The supernatants were assayed for secreted mediators using $\mathrm{PGE}_{2}$ and rat IL-1 $\beta$ immunoassay kits (R\&D Systems, Minneapolis, MN, USA), according to the manufacturer's instructions.

Intracellular ROS assay. Presence of intracellular ROS was measured using a non-fluorescent 2',7'-dichlorofluorescein (DCFH-DA; Molecular Probes) dye as described previously (21). DCF fluorescence was measured using a Wallac 1420 fluorometer (Perkin Elmer, Waltham, MA, USA) at $485 \mathrm{~nm}$ for excitation and $530 \mathrm{~nm}$ for emission.

Western blot analysis. Cells were lysed on ice in lysis buffer [50 mM Tris- $\mathrm{HCl}$ (pH 8.0), $150 \mathrm{mM} \mathrm{NaCl,} 1 \%$ Triton $\mathrm{X}-100,0.5 \%$ sodium deoxycholate, $0.1 \%$ SDS, 1 mM EDTA, $1 \%$ protease inhibitor cocktail and $1 \%$ phosphatase inhibitor cocktail; Sigma]. Following centrifugation, the supernatant was collected and assayed for protein concentration using a DC Protein Assay kit (Bio-Rad, Hercules, CA, USA). Lysate samples containing $30 \mu \mathrm{g}$ of protein were fractionated by SDS-10\% polyacrylamide gel electrophoresis and then electroblotted onto nitrocellulose membranes. The membranes were probed with primary antibodies and immunoreactivity was detected with ECL Reagent (Amersham Biosciences, Piscataway, NJ, USA).

Statistical analysis. For statistical analysis, data were expressed as the mean \pm SEM from three independent experiments. The Student's paired t-test was used for statistical analyses which were performed using SPSS software (version 13.0, SPSS Inc., Chicago, IL, USA). P<0.05 was considered to indicate a statistically significant difference. 
A

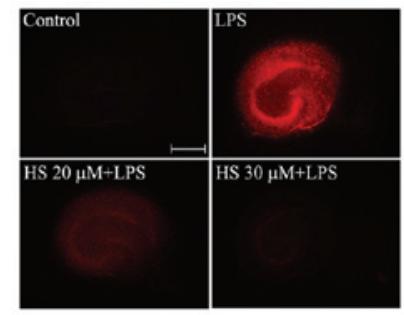

D

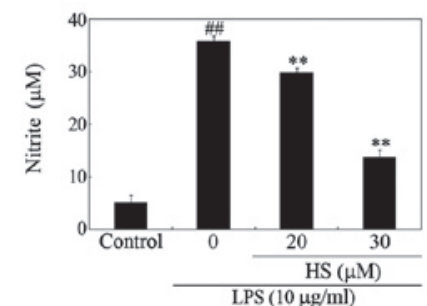

B

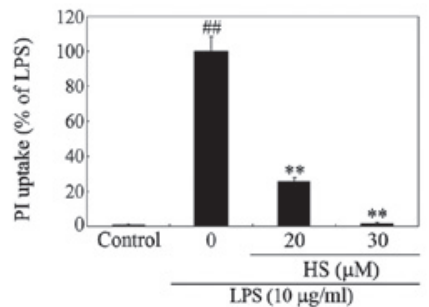

E

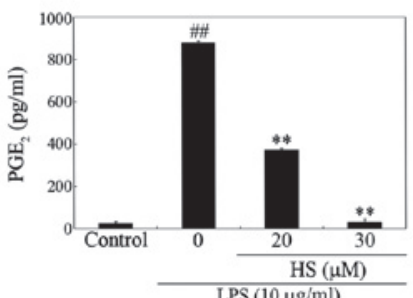

C

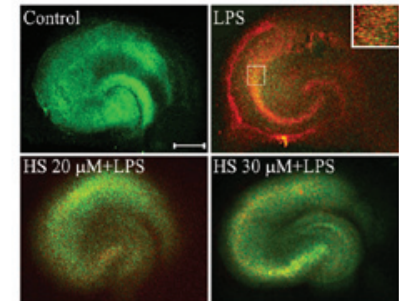

F

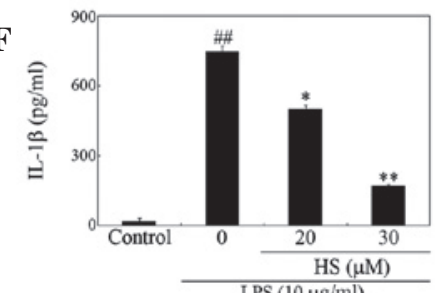

Figure 1. Effect of HS on LPS-induced hippocampal cell death. Organotypic hippocampal slice cultures were pretreated with HS at the indicated concentrations for $30 \mathrm{~min}$ prior to the addition of $10 \mu \mathrm{g} / \mathrm{ml}$ LPS. Following stimulation with LPS for $72 \mathrm{~h}$, the culture medium was replaced with fresh serum-free medium containing PI. (A) PI fluorescence images. Scale bar, $500 \mu \mathrm{m}$. (B) Quantification of PI images. Data are expressed as percentage of the LPS value (mean \pm SEM, $n=10-15$ each). (C) The same slices were immunostained with NeuN that marked neuronal nuclei (NeuN, green; PI uptake, red). A magnified image from an outlined area in the region of the LPS-treated hippocampal slices is demonstrated in the right upper panel to reveal the substantial colocalization of NeuN immunoreactivity and PI fluorescence. Scale bar, $500 \mu \mathrm{m}$. Determinations of (D) nitrite, (E) PGE and $_{2}$ (F) IL-1 13 in culture supernatants of hippocampal slices. ${ }^{\# \#} \mathrm{P}<0.001$ vs. control group; ${ }^{* *} \mathrm{P}<0.001,{ }^{*} \mathrm{P}<0.05$ vs. LPS-only treated group. HS, hirsutine; LPS, lipopolysaccharide; PI, propidium iodide; $\mathrm{PGE}_{2}$, prostaglandin $\mathrm{E}_{2} ; \mathrm{IL}-1 \beta$, interleukin-1 $\beta$.

A

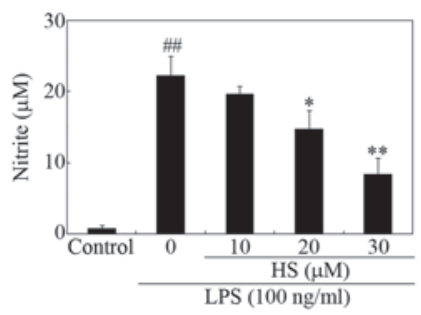

B

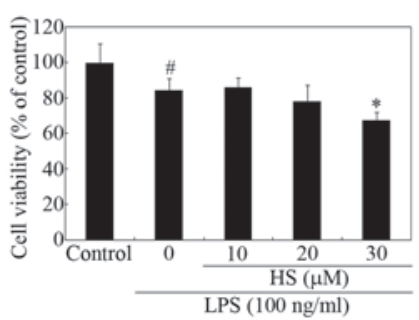

C

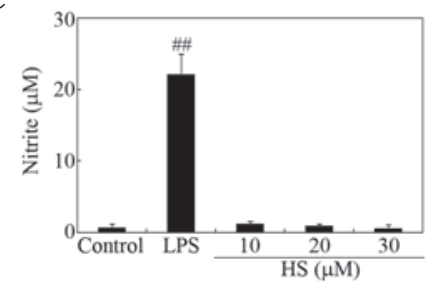

Figure 2. Effect of HS on nitrite production and cell viability in microglial cells. Primary microglial cells were incubated in the absence (control) or presence of LPS. The cells were pretreated with the indicated amounts of HS for 30 min prior to the addition of LPS. Twenty-four hours following LPS treatment, the cultures were subjected to (A) nitrite and (B) cell viability assays. (C) As a reference, cells were treated with HS only for $24 \mathrm{~h}$ and the cultures were subjected to nitrite quantification. Data are expressed as the mean \pm SEM from triplicate assays. ${ }^{\# \#} \mathrm{P}<0.001,{ }^{\|} \mathrm{P}<0.05$ vs. control group; ${ }^{* *} \mathrm{P}<0.001$, ${ }^{*} \mathrm{P}<0.05$ vs. LPS-only treated group. LPS was previously reported to cause activation-induced cell death in microglia (33). Therefore, cell viability, as measured using the MTT assay, was often reduced by LPS. HS, hirsutine; LPS, lipopolysaccharide.

\section{Results}

Protection against LPS-mediated neuronal damage. In an effort to develop neuroprotective drugs, strategies to ameliorate the inflammatory microenvironment, which indirectly damages neurons via glial cell mediators, are promising (4). We previously established an experimental condition to determine the effect of LPS exposure on neuronal damage in organotypic hippocampal slice cultures (20). In the present study, slice cultures exposed to LPS for $72 \mathrm{~h}$ exhibited marked PI uptake in the hippocampus in comparison to untreated control slices (Fig. 1A). The increased PI uptake was markedly blocked by treatment with HS (Fig. 1A and B), in parallel with the inhibition of LPS-induced production of various proinflammatory mediators, including $\mathrm{NO}, \mathrm{PGE}_{2}$ and IL-1 $\beta$ (Fig. 1D-F). Reduced immunoreactivity of NeuN, a neuronal-specific marker, accompanied with elevated PI fluorescence indicated that loss of neurons resulted from the LPS insult (Fig. 1C). Treatment with HS restored immunoreactivity of NeuN and simultaneously decreased PI fluorescence (Fig. 1C). Together, these results indicate that HS has protective effects against inflammation-induced neurotoxicity.

Suppression of LPS-stimulated microglial inflammatory responses. To determine the mechanisms of the anti-inflammatory effects of HS in more detail, various mediators of microglial activation were measured. The effects of treatment with HS on secretion of proinflammatory mediators from microglial cells were tested. HS suppressed LPS-induced nitrite release from microglial cells in a dose-dependent manner (Fig. 2A). Cell viability, as measured using the MTT assay, was not significantly affected at $\leq 20 \mu \mathrm{M}$ (Fig. 2B). HS appeared to decrease cell viability of activated microglia to a degree at $30 \mu \mathrm{M}$ (Fig. 2B), however, it alone had no effect on basal NO release at $<30 \mu \mathrm{M}$ (Fig. 2C). 
A

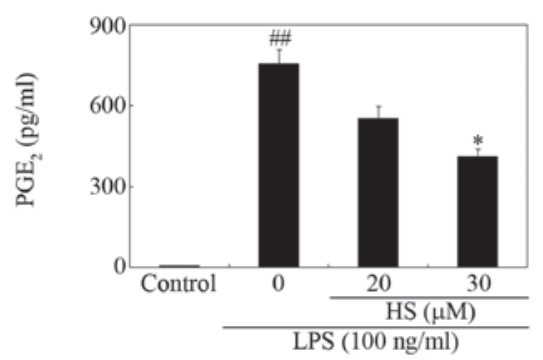

B

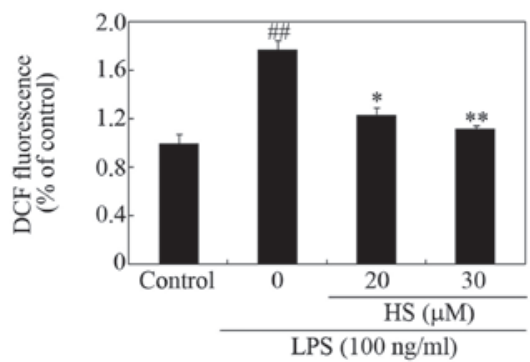

Figure 3. Effect of HS on secretion of $\mathrm{PGE}_{2}$ and production of ROS. Primary microglial cultures were prepared in triplicate and stimulated with LPS $(100 \mathrm{ng} / \mathrm{ml})$ with or without pretreatment with indicated amounts of HS. (A) Following $24 \mathrm{~h}$ incubation, culture supernatants were assayed for $\mathrm{PGE}_{2}$ using ELISA. (B) Following $6 \mathrm{~h}$ LPS incubation with or without pretreatment with HS, levels of intracellular ROS in microglia were determined using DCFH-DA. Data are expressed as the mean \pm SEM from triplicate assays. ${ }^{\# \#} \mathrm{P}<0.001$ vs. control group; ${ }^{* *} \mathrm{P}<0.001,{ }^{*} \mathrm{P}<0.05$ vs. LPS-only treated group. $\mathrm{HS}$, hirsutine; $\mathrm{PGE}_{2}$, prostaglandin $\mathrm{E}_{2}$; ROS, reactive oxygen species; LPS, lipopolysaccharide.

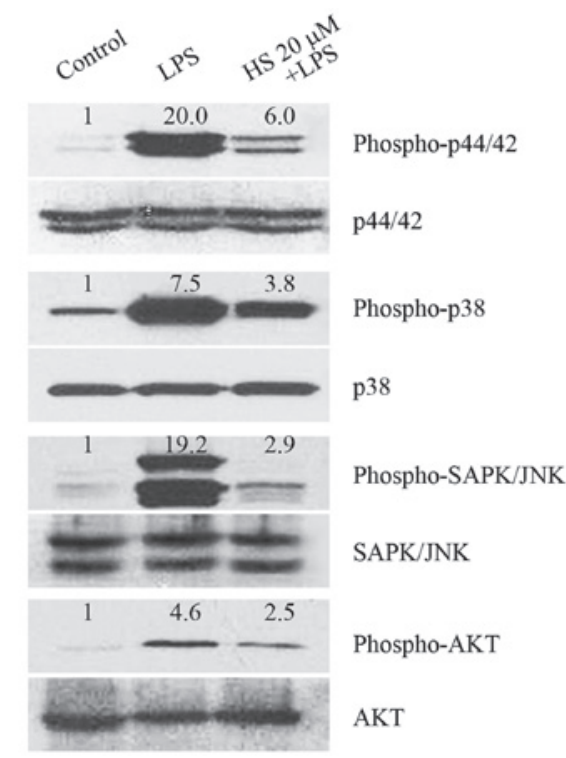

Figure 4. Effect of HS on signaling molecules in LPS-stimulated microglial cells. Primary microglial cells were pretreated with $20 \mu \mathrm{M}$ HS for $30 \mathrm{~min}$ prior to LPS addition. Following stimulation with LPS for $30 \mathrm{~min}$, whole cell lysates were analyzed by western blot analysis. Representative blots from three independent experiments are shown. Band intensities were measured using a densitometer and phosphorylated protein levels were normalized against the total form of each kinase. HS, hirsutine; LPS, lipopolysaccharide.

Effect of HS on secretion of PGE2 and production of $R O S$. HS was found to reduce LPS-induced production of $\mathrm{PGE}_{2}$ (Fig. 3A). Intracellular ROS act as second messengers in the regulation of the LPS-stimulated production of neurotoxic factors in microglia (22). The ROS levels measured using DCFH-DA revealed that pretreatment with HS decreased LPS-induced ROS production in microglia (Fig. 3B).

HS inhibits multiple signaling pathways. Multiple signaling pathways, including those involving mitogen-activated protein kinases (MAPKs) and Akt, have been reported to be involved in LPS-induced signal transduction, which results in the induction of proinflammatory gene expression (23-26). The present study demonstrates that HS markedly inhibited the LPS-enhanced phosphorylation of p44/42 MAPK, p38, SAPK/ JNK (Fig. 4). Among the kinases, phosphorylation of SAPK/ JNK was reduced to the greatest extent by HS (Fig. 4). Taken together, the present data indicate that the anti-inflammatory action of HS in microglia is, at least in part, mediated by the inhibition of these signaling pathways.

\section{Discussion}

In the present study, HS, one of the major alkaloids of $U$. rhynchophylla, effectively repressed diverse inflammatory mediators induced by LPS, including $\mathrm{NO}, \mathrm{PGE}_{2}$, intracellular ROS and phosphorylation of MAPKs and Akt in primary microglial cell culture. Beyond the control of microglial activation, more direct efficacy of HS against inflammation-induced neurotoxicity was observed in hippocampal slice cultures. As such, our results demonstrate that HS may be useful in ameliorating brain disorders associated with uncontrolled microglia-mediated inflammatory responses. Taken together with previous studies on anti-inflammatory actions of rhynchophylline and isorhynchophylline $(27,28)$, the present study supports a pharmacological potential for $U$. rhynchophylla and its active components in manipulating neuroinflammation associated with diverse neuropathologies.

Among the diverse neuroprotective activities of U.rhynchophylla, antioxidant properties are well established $(12,14,29)$. In previous studies, $U$. rhynchophylla exhibited the ability to reduce levels of free radicals in rat brain and increase glutathione levels in PC12 cells $(12,29)$. U. rhynchophylla inhibits the NMDA receptor-activated ion current in hippocampal neurons (10). Additionally, U. rhynchophylla inhibits the aggregation of amyloid $\beta$ protein, a pathological hallmark of Alzheimer's disease (9). Neuroprotective potential conferred by HS has also been explained with its inhibitory capacities on oxidative stress, ion channels and $\mathrm{Ca}^{2+}$ influx $(14,15,30)$. However, little is known regarding the effects of U.rhynchophylla and its active compounds on microglia.

According to a previous study, $U$. rhynchophylla reduces microglial activation in the region of neuronal damage caused by kainic acid administration (13). In the present study, $U$. rhynchophylla reduced ED1- and inducible NO synthaseimmunoreactive cell counts in rat brain, demonstrating that U. rhynchophylla may suppress microglia activation in vivo. Numerous studies have demonstrated an inhibitory function of rhynchophylline-type alkaloids of $U$. rhynchophylla in microglial activation in vitro $(27,28)$. Rhynchophylline and isorhynchophylline have been shown to suppress the release of $\mathrm{NO}$ and proinflammatory cytokines and the phosphorylation of p44/42 and p38 MAPKs in LPS-activated N9 microglial cell lines (28). 
A detailed study on alkaloids from $U$. rhynchophylla previously identified that geissoschizine methyl ether, a corynanthean-type indole alkaloid, is a potent acetylcholinesterase inhibitor and may be a suitable candidate for Alzheimer's disease (31). Matsumoto et al demonstrated that isorhynchophylline regulates neurotransmission by suppressing the serotonin 5-hydroxytryptamine $2 \mathrm{~A}$ receptor function in the brain by competitive antagonism (32). Studies of alkaloids, including the present study, may broaden understanding of the pharmacological value of these compounds of $U$. rhynchophylla in the treatment of neuropathologies, by highlighting various beneficial roles in neuronal survival, synaptic plasticity and microglial activation in the CNS. In this sense, the present results may stimulate further investigation to confirm novel neuropharmacological roles of each individual compound of U. rhynchophylla.

\section{References}

1. Hanisch UK and Kettenmann H: Microglia: active sensor and versatile effector cells in the normal and pathologic brain. Nat Neurosci 10: 1387-1394, 2007.

2. Walter $\mathrm{L}$ and Neumann $\mathrm{H}$ : Role of microglia in neuronal degeneration and regeneration. Semin Immunopathol 31: 513-525, 2009.

3. Sugama S, Takenouchi T, Cho BP, Joh TH, Hashimoto M and Kitani H: Possible roles of microglial cells for neurotoxicity in clinical neurodegenerative diseases and experimental animal models. Inflamm Allergy Drug Targets 8: 277-284, 2009.

4. Skaper SD: The brain as a target for inflammatory processes and neuroprotective strategies. Ann NY Acad Sci 1122: 23-34, 2007.

5. Ehrman TM, Barlow DJ and Hylands PJ: Phytochemical informatics of traditional Chinese medicine and therapeutic relevance. J Chem Inf Model 47: 2316-2334, 2007.

6. Horie S, Yano S, Aimi N, Sakai S and Watanabe K: Effects of hirsutine, an antihypertensive indole alkaloid from Uncaria rhynchophylla, on intracellular calcium in rat thoracic aorta. Life Sci 50: 491-498, 1992

7. Masumiya H, Saitoh T, Tanaka Y, Horie S, Aimi N, Takayama H, Tanaka $\mathrm{H}$ and Shigenobu K: Effects of hirsutine and dihydrocorynantheine on the action potentials of sino-atrial node, atrium and ventricle. Life Sci 65: 2333-2341, 1999.

8. Wu LX, Gu XF, Zhu YC and Zhu YZ: Protective effects of novel single compound, hirsutine on hypoxic neonatal rat cardiomyocytes. Eur J Pharmacol 650: 290-297, 2011.

9. Fujiwara H, Takayama S, Iwasaki K, Tabuchi M, Yamaguchi T, Sekiguchi K, Ikarashi Y, Kudo Y, Kase Y, Arai H and Yaegashi N: Yokukansan, a traditional Japanese medicine, ameliorates memory disturbance and abnormal social interaction with antiaggregation effect of cerebral amyoid $\beta$ proteins in amyloid precursor protein transgenic mice. Neuroscience 180: 305-313, 2011.

10. Lee J, Son D, Lee P, Kim DK, Shin MC, Jang MH, Kim CJ, Kim YS, Kim SY and Kim H: Protective effect of methanol extract Uncaria rhynchophylla against excitotoxicity induced by $\mathrm{N}$-methyl-d-aspartate in rat hippocampus. J Pharmacol Sci 92: $70-73,2003$.

11. Lee SC, Linh PT, Jing Z, Ryu SY, Myung CS, Kim YH and Kang JS: Effects of repeated administration of Uncaria hooks on the acquisition and central neuronal activities in ethanol-treated mice. J Ethnopharmacol 94: 123-128, 2004.

12. Shim JS, Kim HG, Ju MS, Choi JG, Jeong SY and Oh MS Effects of the hook of Uncaria rhynchophylla on neurotoxiciy in the 6-hydroxydopamin model of Parkinson's disease. J Ethnopharmacol 126: 361-365, 2009.

13. Tang NY, Liu CH, Su SY, Jan YM, Hsieh CT, Cheng CY, Shyu WC and Hsieh CL: Uncaria rhynchophylla (Miq) Jack plays a role in neuronal protection in kainic acid-treated rats. Am J Chin Med 38: 251-263, 2010.
14. Kawakami Z, Kanno H, Ikarashi Y and Kase Y: Yokukansan, a kampo medicine, protects against glutamate cytotoxicity due to oxidative stress in PC12 cells. J Ethnopharmacol 134: 74-81, 2011.

15. Shimada Y, Goto H, Itoh T, Sakakibara I, Kubo M, Sasaki H and Terasawa K: Evaluation of the protective effects of alkaloids isolated from the hooks and stems of Uncaria sinensis on glutamate-induced neuronal death in cultured cerebellar granule cells from rats. J Pharm Pharmacol 51: 715-722, 1999.

16. Stoppini L, Buchs PA and Muller D: A simple method for organotypic cultures of nervous tissue. J Neurosci Methods 37: 173-182, 1991.

17. You JM, Yun SJ,Nam KN, Kang C, Won R and Lee EH: Mechanism of glucocorticoid-induced oxidative stress in rat hippocampal slice cultures. Can J Physiol Pharmacol 87: 440-447, 2009.

18. Vornov JJ, Tasker RC and Park J: Neurotoxicity of acute glutamate transport blockade depends on coactivation of both NMDA and AMPA/kainate receptors in organotypic hippocampal cultures. Exp Neurol 133: 7-17, 1995.

19. McCarthy KD and de Vellis J: Preparation of separate astroglial and oligodendroglial cell cultures from rat cerebral tissue. J Cell Biol 85: 890-902, 1980.

20. Nam KN, Son MS, Park JH and Lee EH: Shikonins attenuate microglial inflammatory responses by inhibition of ERK, Akt and NF-kappaB: neuroprotective implications. Neuropharmacology 55: 819-825, 2008.

21. Nam KN, Choi YS, Jung HJ, Park GH, Park JM, Moon SK, Cho KH, Kang C, Kang I, Oh MS and Lee EH: Genipin inhibits the inflammatory response of rat brain microglial cells. Int Immunopharmacol 10: 493-499, 2010

22. Qin L, Liu Y, Wang T, Wei SJ, Block ML, Wilson B, Liu B and Hong JS: NADPH oxidase mediates lipopolysaccharide-induced neurotoxicity and proinflammatory gene expression in activated microglia. J Biol Chem 279: 1415-1421, 2004.

23. Bhat NR, Zhang P, Lee JC and Hogan EL: Extracellular signalregulated kinase and p38 subgroups of mitogen-activated protein kinases regulate inducible nitric oxide synthase and tumor necrosis factor-alpha gene expression in endotoxin-stimulated primary glial cultures. J Neurosci 18: 1633-1641, 1998.

24. Jones BW, Heldwein KA, Means TK, Saukkonen JJ and Fenton MJ: Differential roles of toll-like receptors in the elicitation of proinflammatory responses by macrophages. Ann Rheum Dis 60: 6-12, 2001.

25. Koistinaho M and Koistinaho J: Role of p38 and p44/42 mitogenactivated protein kinases in microglia. Glia 40: 175-183, 2002.

26. Lee YG, Lee J, Byeon SE, Yoo DS, Kim MH, Lee SY and Cho JY: Functional role of Akt in macrophage-mediated innate immunity. Front Biosci 16: 517-530, 2011.

27. Yuan D, Ma B, Wu C, Yang J, Zhang L, Liu S, Wu L and Kano Y: Alkaloids from the leaves of Uncaria rhynchophylla and their inhibitory activity and NO production in lipopolysaccharideactivated microglia. J Nat Prod 71: 1271-1274, 2008.

28. Yuan D, Ma B, Yang J, Xie Y, Wang L, Zhang L, Kano Y and Wu C: Anti-inflammatory effects of rhynchophylline and isorhynchophylline in mouse N9 microglial cells and the molecular mechanism. Int Immunopharmacol 9: 1549-1554, 2009.

29. Hsieh CL, Tang NY, Chiang SY, Hsieh CT and Lin JG: Anticonvulsive and free radical scavenging actions of two herbs, Uncaria rhynchophylla (Miq) Jack and Gastrodia elata B1., in kainic acid-treated rats. Life Sci 65: 2071-2082, 1999.

30. Nakazawa K, Watano T, Ohara-Imaizumi M, Inoue $K$, Fujimori K, Ozaki Y, Harada M and Takanaka A: Inhibition of ion channels by hirsutine in rat pheochromocytoma cells. Jpn J Pharmacol 57: 507-515, 1991.

31. Yang ZD, Duan DZ, Du J, Yang MJ, Li S and Yao XJ: Geissoschizine methyl ether, a corynanthean-type indole alkaloid from Uncaria rhynchophylla as a potential acetylcholinesterase inhibitor. Nat Prod Res 26: 22-28, 2012.

32. Matsumoto K, Morishige R, Murakami Y, Tohda M, Takayama H, Sakakibara I and Watanabe H: Suppressive effects of isorhynchophylline on 5-HT2A receptor function in the brain: behavioural and electrophysiological studies. Eur J Pharmacol 517: 191-199, 2005.

33. Lee P, Lee J, Kim S, Lee MS, Yagita H, Kim SY, Kim H and Suk K: NO as an autocrine mediator in the apoptosis of activated microglial cells: correlation between activation and apoptosis of microglial cells. Brain Res 892: 380-385, 2001. 